\title{
Starvation-responsive glycine-rich protein gene in the silkworm Bombyx mori
}

\author{
Kiyoko Taniai $\cdot$ Chikara Hirayama $\cdot$ Kazuei Mita \\ Kiyoshi Asaoka
}

Received: 28 February 2014 / Revised: 18 June 2014 / Accepted: 9 July 2014 / Published online: 7 August 2014

(C) The Author(s) 2014. This article is published with open access at Springerlink.com

\begin{abstract}
Four glycine-rich protein (GRP) genes were identified from expressed sequence tags of the maxillary galea of the silkworm. All four genes were expressed in the maxillary pulp, antenna, labrum, and labium, but none of the genes were expressed in most internal organs. Expression of one of the genes, termed bmSIGRP, was further increased approximately fivefold in the mouth region (including the maxilla, antenna, labrum, labium, and mandible) after $24 \mathrm{~h}$ of starvation. bmSIGRP expression peaked at $24 \mathrm{~h}$ and gradually declined during the subsequent 2 days. When a synthetic diet not containing proteins was fed, bmSIGRP expression increased significantly in the
\end{abstract}

Communicated by I.D. Hume.

Electronic supplementary material The online version of this article (doi:10.1007/s00360-014-0846-8) contains supplementary material, which is available to authorized users.

K. Taniai $(\bowtie) \cdot$ K. Asaoka

Division of Insect Sciences, National Institute of Agrobiological

Sciences, 1-2 Owashi, Tsukuba, Ibaraki 305-8634, Japan

e-mail: taniai@affrc.go.jp

C. Hirayama

Genetically Modified Organism Research Center, National Institute of Agrobiological Sciences, 1-2 Owashi, Tsukuba, Ibaraki 305-8634, Japan

K. Mita

Agrogenomics Research Center, National Institute of Agrobiological Sciences, 1-2 Owashi, Tsukuba, Ibaraki 305-8634, Japan

Present Address:

K. Mita

State Key Laboratory of Silkworm Genome Biology, Southwest University, Tiansheng Road \#1, Beibei, Chongqing 400716,

China mouth region to levels similar to that observed in starved larvae. Synthetic diets that lacked vitamins or salts but contained amino acids did not significantly affect $b m S I G R P$ expression. These results suggest that amino acid depletion increases $b m S I G R P$ expression.

Keywords Bombyx mori - Glycine-rich protein gene . Starvation $\cdot$ Amino acid depletion

\section{Introduction}

Glycine-rich proteins (GRPs) with quasi-repetitive glycinerich (GR) motifs $\left(\mathrm{Gly}_{\mathrm{n}}-\mathrm{X}_{1-2}\right)$ have been widely identified in prokaryotes and eukaryotes. These GRPs share similar GR motifs, but their characteristics and functions are diverse. GRPs have been found to act as structural proteins, antimicrobial proteins (Baba et al. 1987; Axén et al. 1997; Park et al. 2000), RNA-binding proteins (Cobianchi et al. 1994; Sato 1994), or stress-inducible proteins. Structural proteins with GR motifs have been identified as components of the body surface, including keratins and loricrins of mammalian skin (Mehrel et al. 1990), chorion and egg-shell proteins of invertebrates (Hamodrakas et al. 1985; Sudo et al. 1997), insect cuticular proteins (Højrup et al. 1986; Charles et al. 1992; Andersen et al. 1995), cement proteins of ticks (Maruyama et al. 2010), and plant cell-wall proteins (Keller 1993). In the silkworm Bombyx mori, the expression of several GRP genes predicted to encode cuticular proteins was identified in epidermal cells (Suzuki et al. 2002; Zhong et al. 2006). Stress-inducible GRPs are extensively documented in plants. Plant GRPs encompass five classes based on their primary structures (Mousavi and Hotta 2005; Mangeon et al. 2010). Each class contains several GRPs that are transcriptionally regulated by different types of stresses 
and factors, such as water stress, wounding, infection, cold shock, abscisic acid treatment, and salicylic acid treatment (Sachetto-Martins et al. 2000). Three cuticular GRP genes have been identified in an insecticide-resistant strain of the Colorado potato beetle Leptinotarsa decemlineata (Say); all are strongly induced by insecticides (Zhang et al. 2008).

In this study, we identified four GRP genes from expressed sequence tags (ESTs) of maxillary galea from silkworm, and found that one is strongly induced by starvation and amino acid depletion. To the best of our knowledge, this is the first GRP gene to be identified as being inducible by nutritional stress in insect.

\section{Materials and methods}

\section{Identification and characterization of $G R P$ genes}

The EST library of the maxillary galea (fmxg-EST) of 5thinstar B. mori larvae was previously described (Yoshizawa et al. 2011). The GRP genes were manually identified in the library. The signal peptide was predicated using the SignalP 4.1 program (http://www.cbs.dtu.dk/services/SignalP/), and the isoelectric point and molecular size were predicated using the GENETYX-MAC software (version 14; GENETYX Co, Tokyo). The genome location of the gene was determined through a scaffold sequence search of the KAIKObase database (http://kaikoblast.dna.affrc.go.jp).

\section{Insects and diet}

The B. mori strains $\mathrm{N} 137 \times \mathrm{C} 146$ and $\mathrm{N} 601 \times \mathrm{C} 601$ were reared at $25{ }^{\circ} \mathrm{C}$ under a photoperiod of $12 \mathrm{~h}$ light/12 h dark and fed the Silkmate 2S and Silkmate L4-M artificial diets, respectively. The Silkmate $2 \mathrm{~S}$ diet contains a high amount of mulberry leaves, whereas the Silkmate L4-M diet contains only a few percent mulberry leaves (the details of the recipe are not indicated by the manufacturer, Nippon Nosan Co. Ltd.). The N601 × C601 strain is relatively polyphagous and eats the L4-M diet. For nutrition deprivation analyses, the N601 × C601 larvae were transferred from the L4-M diet to a synthetic diet (Supplemental Table 1) established by Yanagawa et al. (1995) after the fourth molt. The synthetic diet, designated SAD100 (Synthetic Artificial Diet $100 \%$ ), contains all of the nutrients necessary for larval growth. To prepare the diets lacking amino acids (SAD-AA), inorganic salts (SADIS), and vitamins (SAD-V), an equivalent amount (weight) of cellulose powder was added in place of the deficient nutrients.

\section{Stress test}

Five 1-day-old fifth-instar larvae were placed in a plastic cup (12 cm in diameter) with the Silkmate $2 \mathrm{~S}$ diet. The cup was then covered with a lid and placed at $25{ }^{\circ} \mathrm{C}$ and 60 $65 \%$ humidity; these conditions were considered the control conditions. To test starvation, larvae were placed in an empty cup with a lid. To test low- and high-humidity conditions, larvae in a cup (without a lid) were placed at $25^{\circ} \mathrm{C}$ in a desiccator chamber, which was adjusted to $11 \%$ or $84 \%$ humidity using a saturated $\mathrm{LiCl}$ or $\mathrm{KCl}$ solution, respectively. The humidity was confirmed using a hygrometer. After $24 \mathrm{~h}$ of treatment, the larval mouth region containing all of the oral appendages (antennae, maxilla, labrum, labium, and mandible) was removed and analyzed by quantitative reverse transcription-polymerase chain reaction (qRT-PCR).

\section{qRT-PCR}

Tissues were removed from 1- to 2-day-old fifth-instar larvae, immediately homogenized in $1 \mathrm{ml}$ of cold ISOGEN reagent (Nippon Gene) using a polypropylene pestle, and maintained at $-80{ }^{\circ} \mathrm{C}$ until use. Total RNA was separated from the DNA, proteins, and other substances according to the manufacturer's instructions. cDNA was synthesized from $1 \mu \mathrm{g}$ of total RNA using a Transcriptor First-Strand cDNA Synthesis Kit (Roche) with an anchored oligo(dT) ${ }_{18}$ primer. qRT-PCR analysis was performed in a $20-\mu \mathrm{l}$ reaction volume containing $0.5 \mu \mathrm{M}$ primers, $1 \times$ LightCycler 480 SYBR Green I Master Mix, and $4 \mu$ of the template sample or a standard plasmid. The standard plasmids were prepared by amplifying the coding regions of the GRP genes by PCR and inserting them into the pGEM-T Easy plasmid (Promega). Standard curves were generated using the signals from serial dilutions $(0.1-1,000 \mathrm{pg})$ of the plasmids, and the crossing points of the standards and the samples were used to determine the amount of transcript in each sample. PCR was conducted using the following temperature program in a Light Cycler 480 Real-Time PCR System (Roche): $95{ }^{\circ} \mathrm{C}$ for $5 \mathrm{~min}$ followed by 45 cycles at $95{ }^{\circ} \mathrm{C}$ for $5 \mathrm{~s}, 58{ }^{\circ} \mathrm{C}$ for $5 \mathrm{~s}$, and $72{ }^{\circ} \mathrm{C}$ for $10 \mathrm{~s}$. Following the PCR, the absence of unwanted by-products was confirmed through melting curve analysis. The amount of transcripts was normalized to the amount of the actin $A 3$ gene (Mounier and Prudhomme 1986) in each sample, which was analyzed using the TaqMan system as described previously (Yoshizawa et al. 2011). The primers used in the experiments are listed in Supplemental Table 2.

Quantification of amino acids in hemolymph

The concentration of alpha-amino groups in the hemolymph was measured through the Ninhydrin reaction (Yemm and Cocking 1954). The hemolymph was collected by cutting the abdominal legs on ice and removing the hemocytes in the hemolymph by centrifugation at $3,000 \times g$ 


\begin{abstract}
BmSIGRP mnifyyitvlicaclayvsaLPSAIAGAEDLQPAESRWNHGGGHHGGGHHGGGHHGGGHYGGGHHGGHGGYKGGYGHGR (59)
Fmxg01107 mykstaiiffvvmmvgsslqQGGAEGGTNAGIGFGANAGGGAGSDAGAGAGGGAGFOFGANAGAGGGGEAGSSNGFGK (58)

Fmxg10C13 marlaipalalglfacacaAPGYGGGSGSLGGGSGGGGGFGGGGGGGFGGGGGKGGYGGGSHGGDSGGSHGGGGGSH GGGGGFGSGGGGGSGGGGGGSHGGGGGLGSGVGGGSGGGGGGSHGGGGGSHGGGSGSHGGGGGSHGGSGGE GSGGGSGHGGGGGFGGGSGGGNGGGFGGGHGGGGGGGGGGGHGGGGYGGHKGGYGR (185)

Fmxg01D19 mghggvvvsflalaavasaSIGDGDNSLDGGERVVLVAPVLAPPRDSRTHVTDRARQFPILVLLAQPQISDYRPEPSAKSLKGHL EPIYVQKLEDQENNKGTLNRQKRTLFFGLKGYRKGHHGYDRGGGYGGGGGYGGGGGYGGGGGYGGGGGYGGGGGY GGGSGYGGGGGHGGGYGGGGGHGGGYGGNOGNYGYPTGCGGGCGGGOGSAIAIATANG (200)
\end{abstract}

Fig. 1 Deduced amino acid sequences of the four GRP genes. The small letters indicated in boxes correspond to the predicted signal peptides. Underlined letters depict GR motifs. Numbers in parentheses indicate the number of amino acids in the predicted mature proteins

Table 1 Characteristics of the deduced mature protein of four GRP genes

$M W$ molecular weight, $\mathrm{pI}$ isoelectric point, $C h$ chromosome

\begin{tabular}{lrllrll}
\hline Protein name & $\begin{array}{l}\text { MW } \\
(\mathrm{kDa})\end{array}$ & $\mathrm{p} I$ & Gly $(\%)$ & Ch & $\begin{array}{l}\text { Hydrophobic } \\
\text { residues }(\%)\end{array}$ & Acc. No. \\
\hline BmSIGRP & 5.8 & 8.21 & 40.7 & 19 & 57.63 & AB839176 \\
Fmxg10C13 & 13.7 & 9.77 & 71.4 & 5 & 75.86 & AB839178 \\
Fmxg01I07 & 4.7 & 3.87 & 44.8 & 16 & 77.30 & AB839177 \\
Fmxg01D19 & 19.4 & 9.14 & 35.6 & 5 & 58.79 & AB839179 \\
\hline
\end{tabular}

for $10 \mathrm{~min}$. The proteins in the supernatant were precipitated through the addition of four volumes of $10 \%$ trichloroacetic acid (TCA), incubating on ice for $15 \mathrm{~min}$, and centrifuging at $14,000 \times g$ for $10 \mathrm{~min}$. To remove the TCA, the supernatant was extracted with an equal volume of diethyl ether. The hemolymph samples were maintained at $-30{ }^{\circ} \mathrm{C}$ until use. The samples were then mixed with $0.2 \mathrm{M}$ citric acid ( $\mathrm{pH}$ 5.0, at a volumetric ratio of 1:9) and then with an equal volume of the Ninhydrin test solution (Wako). The mixture was then boiled for $15 \mathrm{~min}$ and immediately cooled on ice. The solution was mixed with five volumes of $50 \%$ ethanol, and the absorbance at $570 \mathrm{~nm}$ was measured. A standard curve was prepared using varying concentrations of an arginine solution $(0.1,0.5,1$, and $5 \mathrm{mM})$. The experiments were performed three times, and the significance of differences between SAD-AA and SAD100, and between SAD-AA and starvation, was estimated by the Student's $t$ test with $P<0.01$ accepted as significant.

\section{Results}

Identification of $G R P$ genes

We identified four GRP genes, bmSIGRP, fmxg10C13, fmxg01107, and fmxg01D19, in the fmxg-EST library, which was constructed using the $\mathrm{N} 137 \times \mathrm{C} 146$ strain. The frequencies of the four GRP genes in the fmxg-ESTs were $173(4.0 \%), 73(1.7 \%), 48(1.0 \%)$, and $32(0.7 \%)$, respectively, in a total of 4,267 reads, indicating that each is highly expressed in the maxillary galea. Two of the genes, bmSIGRP and fmxg01D19, were previously annotated as cuticular GRP genes (cpg36 and cpg10, respectively) (Futahashi et al. 2008), but evidence was not provided that these genes are cuticular protein genes. The other two genes, fmxg10C13 and fmxg01107, were identified de novo in this study. All four GRP genes encode GRPs with a typical GR motif of a $\left(\mathrm{Gly}_{2-7}-\mathrm{X}_{1-2}\right)$ repeat sequence in the glycine-rich regions and contain a predicted signal peptide (Fig. 1). The characteristics of the deduced mature proteins, including molecular size, $\mathrm{pI}$, and glycine content, vary (Table 1). The sequences of the deduced BmSIGRP and Fmxg01D19 proteins lack the GR motif at the $\mathrm{N}$ terminus, whereas those of Fmxg01I07 and Fmxg10C13 contain the GR motif throughout the protein. In particular, the glycine content of Fmxg10C13 is extremely high (71.4\%). The predicted $\mathrm{p} I$ of three of the GRPs is basic, whereas that of Fmxg01I07 is acidic (3.87). These differences in the $\mathrm{p} I$ value are due to the different $\mathrm{X}$ residues in the GR motifs: the $\mathrm{X}$ residues in BmSIGRP are mostly His, the $\mathrm{X}$ residues in Fmxg01I07 vary (Ala, Phe, Asn, Ser, and Glu), the $\mathrm{X}$ residues in Fmxg10C13 are mostly Phe and His, and the $\mathrm{X}$ residues in Fmxg01D19 are mostly Tyr and His. The locations of the four GRP genes in the genome also differ (Table 1). bmSI$G R P$ was mapped to chromosome 19, fmxg01D19 and fmxg10C13 were mapped to chromosome 5, and fmxg01107 


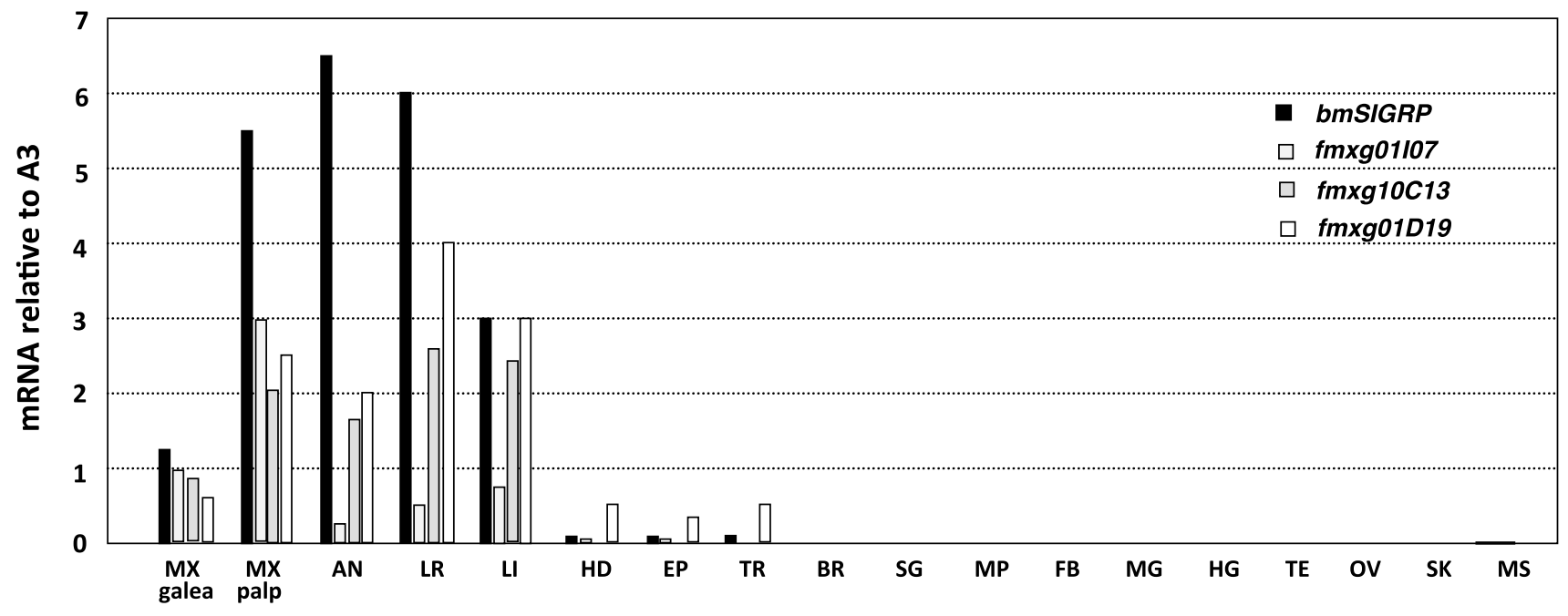

Fig. 2 Distribution of the expression of the four $G R P$ genes. Organs were removed from 5-10 larvae on days 1-2 of the fifth instar, and levels of the transcript corresponding to each of the four GRP genes were quantified by qRT-PCR. The data represent the means of independent experiments $(n=2-5)$. The expression of the actin $A 3$ gene

was mapped to chromosome 16 . These quite different characteristics suggest that the four GRPs have different roles.

\section{Distribution of the four GRP genes}

The expression of the four GRP genes was investigated in the oral appendages of fifth-instar larvae of the N137 × C146 strain: the maxillary galea, maxillary pulp, antenna, labrum and labium, the head lacking all of the previously listed oral appendages, epidermis, trachea, and various internal organs (Fig. 2). All of the four genes were found to be highly expressed in all of the oral appendages. Three of the genes, bmSIGRP, fmxg01107, and fmxg01D19, were weakly expressed in the head, trachea, and epidermis. However, none of the four genes was expressed in the other internal organs, such as the Malpighian tube, fat body, midgut, hindgut, testis, ovary, silk gland, or muscle.

\section{Starvation increases bmSIGRP expression}

We used the N137 $\times$ C146 strain to examine whether the four $G R P$ genes are responsive to stress because many plant $G R P$ genes are regulated by stress. Immediately after the fourth molt, larvae were starved or exposed to low or high humidity for $24 \mathrm{~h}$, and then the mouth region was analyzed for GRP gene expression. Two odorant-binding protein genes (bmobpL1 and bmobpL2) that are stably expressed in the mouth region (Yoshizawa et al. 2011) were used as negative controls. Three GRP genes, fmxg10C13, fmxg01107, and fmxg01D19, were not significantly changed by any of these stresses, similar to the control genes. In contrast, was used as an internal control. $M X$ maxilla, $A N$ antenna, $L R$ labrum, $L I$ labium, $H D$ head without the oral appendages and eyes, $E P$ epidermis of the body, $T R$ trachea, $B R$ brain, $S G$ subesophageal ganglion, $M P$ malpighian tube, $F B$ fat body, $M G$ midgut, $H G$ hindgut, $T E$ testis, $O V$ ovary, $S K$ silk gland, $M S$ muscle

the expression of $b m S I G R P$ increased approximately fivefold in response to starvation (Fig. 3). Neither high nor low humidity affected bmSIGRP expression. Thus, we named the gene bmSIGRP (Bombyx mori Starvation Inducible Glycine-Rich Protein gene). We next analyzed an expression time course of bmSIGRP after starvation. To ensure that the start time of the starvation was the same relative to the larval stage under all conditions, the larvae were fed for $18 \mathrm{~h}$ after the fourth molt, after which either starvation was begun or feeding was continued for 3 days. The mouth region was collected from three to five larvae at each time point, at 3,6,12, 24, 48, and $72 \mathrm{~h}$ after the start of starvation. The PCR results revealed that the expression of $b m S I-$ $G R P$ increased rapidly, reached a maximum at $24 \mathrm{~h}$, and then declined gradually in the subsequent 2 days (Fig. 4).

\section{Amino acid depletion increases bmSIGRP expression}

To determine the factor(s) involved in the increased bmSIGRP expression induced by starvation, we prepared several synthetic diets lacking a particular nutritional component. The complete synthetic diet contained no proteins, but instead contained 18 amino acids. None of the larvae, even those of the relatively polyphagous B. mori strain $(\mathrm{N} 601 \times \mathrm{C} 601)$, ate the diet that lacked sucrose. Therefore, we could not examine the effect of sugar depletion on bmSIGRP expression. In contrast, the larvae ate the diet lacking amino acids (SAD-AA), and their body weights increased equivalently to the larvae that ate the normal artificial diet (L4-M) or the complete synthetic diet (SAD100) (Fig. 5). The larvae also ate the 


\section{Induction fold of relative mRNA}

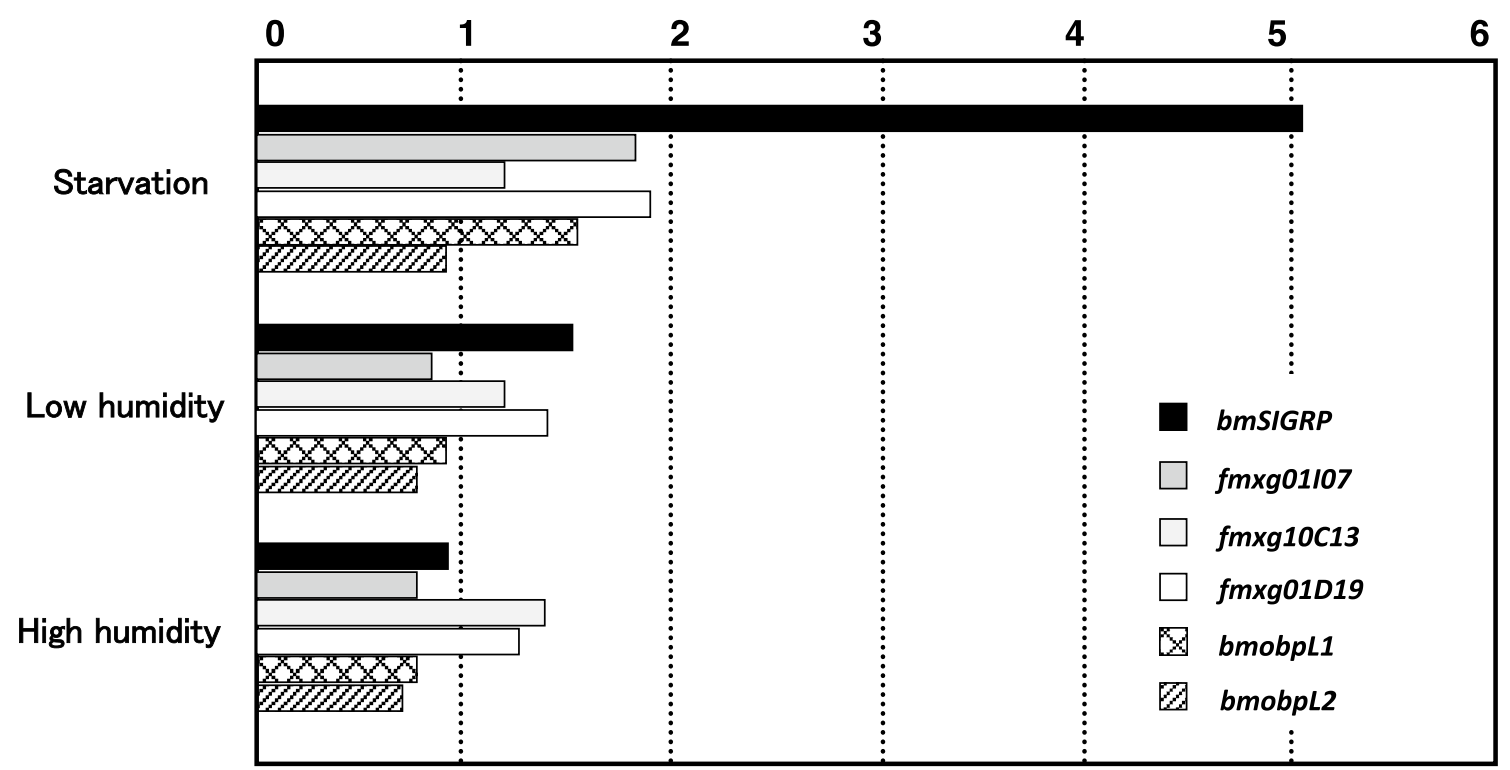

Fig. 3 Starvation increases bmSIGRP expression. After the fourth ecdysis, the larvae were starved or placed under low humidity (11\%) or high humidity (84\%) conditions for $24 \mathrm{~h}$, and the expression of bmSIGRP in the mouth region was analyzed by qRT-PCR. The expression was normalized using the actin $A 3$ gene as an internal con- trol. Induction folds were calculated based on the expression level in control larvae that were reared on L4-M diet at $25{ }^{\circ} \mathrm{C}$ and $60-65 \%$ humidity. Ten larvae were used for each experiment; bars represent the mean values of two experiments. bmobpL1 and bmobpL2 are odorant-binding protein genes that were used as negative controls diet lacking inorganic salts (SAD-IS) and the diet lacking vitamins (SAD-V), although they consumed less than with the L4-M and SAD-100 diets. After $24 \mathrm{~h}$ of diet consumption, bmSIGRP expression was significantly increased in the mouth region of larvae that ate the SADAA diet, equivalent to the $b m S I G R P$ expression increase observed in starved larvae. The other diets did not significantly affect $b m S I G R P$ expression. Thus, amino acid depletion increases the expression of bmSIGRP.

We then analyzed the effect of starvation on the amino acid concentration in the larval hemolymph. Hemolymph was collected at $0,6,12,24$, and $36 \mathrm{~h}$ after the fourth ecdysis from starved N601 × C601 larvae or N601 × C601 larvae fed L4-M, and the concentration of alpha-amino groups was determined using the Ninhydrin reaction. The total alpha-amino group concentration in the hemolymph was higher at $12 \mathrm{~h}$ after the start of feeding, and the level was maintained for the next $24 \mathrm{~h}$. In contrast, the total alphaamino group concentration had decreased in hemolymph of the starved larvae, both female and male, at $12 \mathrm{~h}$ after the fourth ecdysis, and the level remained low during the next $24 \mathrm{~h}$ (Fig. 6a). These results indicate that starvation affects the amino acid concentrations in the hemolymph. We next compared the amino acid concentration in the hemolymph of both male and female larvae that were fed SAD100, SAD-AA, or L4-M, or starved for $24 \mathrm{~h}$ after the fourth ecdysis. The amino acid concentration in larvae that were fed SAD100 was equivalent to that in larvae that were fed L4-M, but significantly lower $(P<0.01)$ in larvae that were fed SAD-AA (Fig. 6b). The concentration in larvae that were fed SAD-AA was even lower than that in starved larvae. These results indicate that both starvation and amino acid depletion result in a decrease in the amino acid concentration in the hemolymph.

\section{Discussion}

Several stress-responsive GRPs with the GR motif have been identified, mainly in plants (Sachetto-Martins et al. 2000). A few beetle cuticular GRP genes that are induced by an insecticide are thought to provide strength to the beetle's cuticle and therefore help it survive under severe environmental conditions (Zhang et al. 2008). We identified four GRP genes that are highly expressed in oral appendages of the silkworm (Fig. 2). This expression pattern suggests that these GRPs are related to feeding behavior or to the complex structure of the oral appendages. Although the distribution of the expression of these four GRP genes is similar, the functions of the genes may be different because the deduced proteins differ in characteristics such as molecular size, $\mathrm{p} I$, and amino acid composition. We 


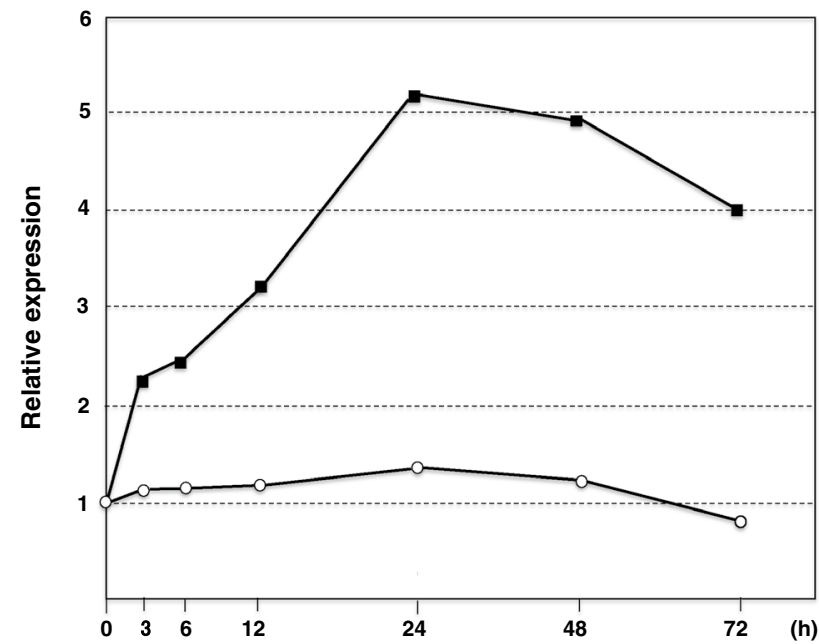

Fig. 4 Expression time course of bmSIGRP. After the fourth ecdysis, larvae were fed an artificial diet for $18 \mathrm{~h}$ and then fed or starved for 3 days. The mouth region was collected from three larvae at the indicated time points, and the $b m S I G R P$ transcript was quantified by qRT-PCR. Expression of the actin $A 3$ gene was used as an internal control

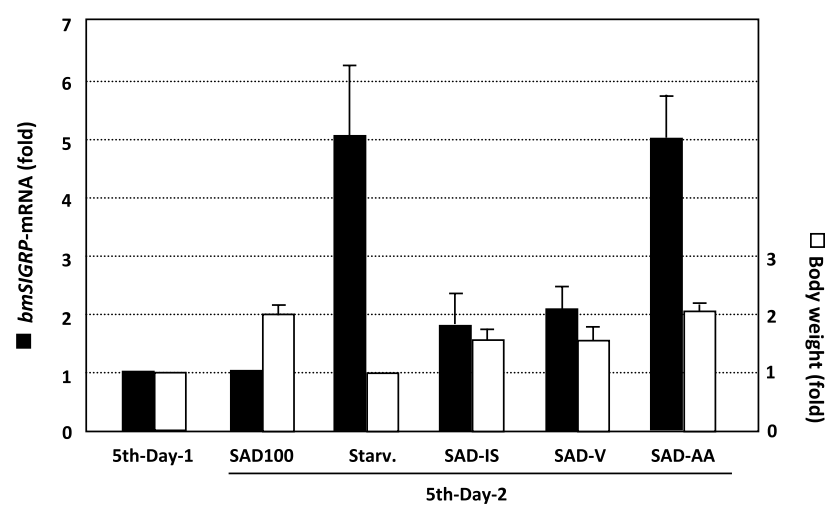

Fig. 5 Amino acid depletion increases bmSIGRP expression. After the fourth ecdysis, larvae were fed experimental diets (SAD100, SAD-IS, SAD-V, or SAD-AA) or starved (Starv.) for $24 \mathrm{~h}$. The $b m S I-$ $G R P$ transcript was quantified by qRT-PCR. Expression of the actin $A 3$ gene was used as an internal control. Black bars represent the relative levels of $b m S I G R P$ mRNA in the mouth region induced after each treatment. White bars indicate the fold increase in body weight. Error bars indicate the standard deviations for three independent experiments

examined whether some environmental stresses regulate the four GRP genes and found that one of the genes, $b m S I$ $G R P$, shows significantly increased expression after starvation (Fig. 3). In contrast, the expression of the other three GRPs was not altered by starvation. Many GRP genes have been reported to be upregulated by dryness (Sachetto-Martins et al. 2000). However, environmental humidity stress did not affect bmSIGRP expression, suggesting that water depletion caused by starvation is unrelated to bmSIGRP
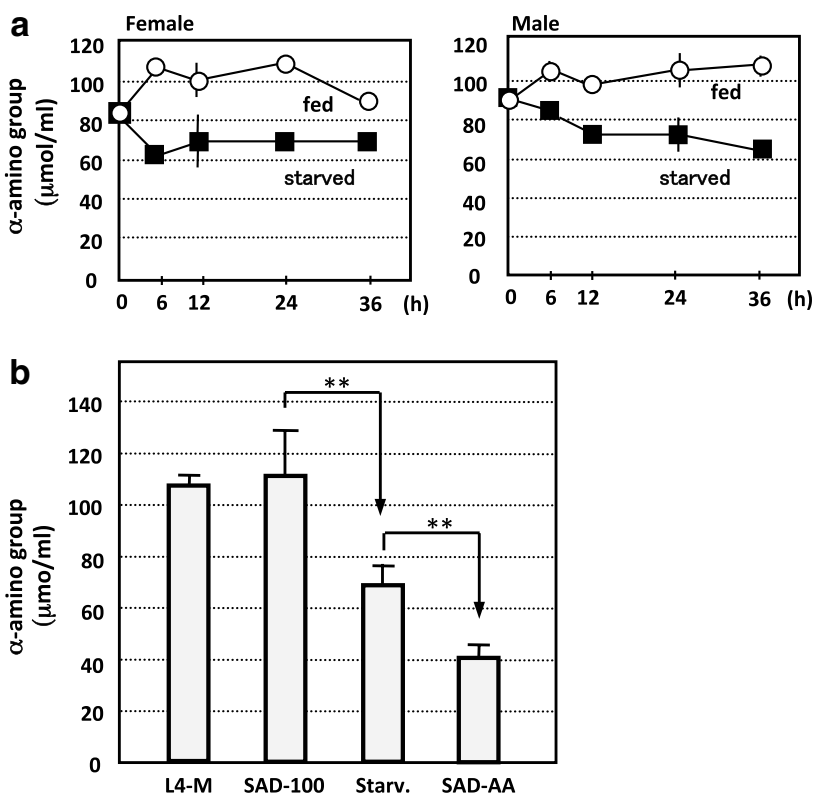

Fig. 6 Amino acid concentration in larval hemolymph. a Concentration of alpha-amino groups in the hemolymph determined by the Ninhydrin reaction. Hemolymph was collected from larvae at 0,6 , 12,24 , and $36 \mathrm{~h}$ after initiation of feeding of the L4-M diet (white circles) or of starvation (black squares) immediately after the fourth ecdysis. Vertical bars on the symbols indicate the standard error from three independent experiments, and symbols without bars indicate that the standard error is too small to be depicted in the figure. b Concentration of alpha-amino groups in the hemolymph of larvae (mix of female and male) at $24 \mathrm{~h}$ after the fourth ecdysis. Standard errors were calculated from three independent experiments. The double stars between SAD100 and Starv. (starvation), and between Starv. and SAD-AA, indicate significant differences $(P>0.01)$ as determined through a $t$ test of data from three independent experiments

regulation. We next examined the effects of nutritional depletion stress on bmSIGRP expression using synthetic diets. If larvae are reared on a synthetic diet beginning as neonates, their growth speed becomes slow and body sizes become diverse. Therefore, a normal artificial diet was used to grow the larvae until the end of the fourth instar. The larvae were then transferred to the synthetic diet after the fourth ecdysis to test nutritional stress. The possibility exists that simply a change in diet causes nutritional stress in the larvae. However, this possible stress did not affect bmSIGRP expression because the bmSIGRP transcript levels were equivalent in larvae that were reared continuously on the L4-M diet and those that were reared on the SAD100 diet for $24 \mathrm{~h}$. Using several different synthetic diets that lack a particular nutrient, we found that amino acid depletion strongly induced $b m S I G R P$ expression to a level similar to that of starvation (Fig. 5). Thus, bmSIGRP is regulated by amino acid availability.

Regulation of bmSIGRP expression might be mediated by the taste organs in the mouth region, the amino acid 
concentration in the hemolymph, or the amino acid concentration in cells. Of these possibilities, we examined whether the amino acid concentration in the hemolymph was related to bmSIGRP expression. Silkworms require ten essential amino acids for normal growth, and deprivation of one of these amino acids rapidly decreases their total protein synthesis (Horie and Inokuchi 1978). If protein synthesis is ceased, the amino acid concentration in the hemolymph may not change. To clarify this issue, we analyzed the concentration of amino acids in the hemolymph during starvation or amino acid depletion. The amino acid concentration decreased in both larvae that were starved and those that were depleted of amino acids after 24 h (Fig. 6a). Notably, the concentration was significantly lower $(P<0.01)$ in larvae that were fed SAD-AA than in starved larvae (Fig. 6b), most likely because the larvae that were fed the SAD-AA diet used energy for the feeding activity, resulting in a higher rate of consumption of the amino acids in the hemolymph in comparison to the starved larvae. These results suggest that the amino acid concentration in the hemolymph is related to the mechanism of $b m S I G R P$ gene regulation.

Depletion of amino acids downregulates the target of the rapamycin (TOR) signaling pathway (Wullschleger et al. 2006), which is widely conserved from yeast to mammals (Colombani et al. 2003; Goberdhan et al. 2005). Because a TOR gene has been isolated from $B$. mori (Zhou et al. 2010), determining whether the regulation mechanism of $b m S I G R P$ is related to regulation mechanism of the TOR signaling pathway may be possible. In addition, identifying the function of bmSIGRP is important. Predicting the function of BmSIGRP based on those of other similar proteins is difficult because GRPs have very diverse functions (Mangeon et al. 2010). To elucidate the function of $b m S I G R P$, attempts to overexpress bmSIGRP in baculovirus will be useful, and knocking out bmSIGRP using a TALEN system, a recently established genetic tool in insects (Ma et al. 2012; Takasu et al. 2013), will be interesting.

Open Access This article is distributed under the terms of the Creative Commons Attribution License which permits any use, distribution, and reproduction in any medium, provided the original author(s) and the source are credited.

\section{References}

Andersen SO, Højrup P, Roepstorff P (1995) Insect cuticular proteins. Insect Biochem Mol Biol 25:153-176

Axén A, Carlsson A, Engström A, Bennich H (1997) Gloverin, an antibacterial protein from the immune hemolymph of Hyalophora pupae. Eur J Biochem 247:614-619

Baba K, Okada M, Kawano T, Komano H, Natori S (1987) Purification of sarcotoxin III, a new antibacterial protein of Sarcophaga peregrina. J Biol Chem 102:69-74
Charles JP, Bouhin H, Quennedey B, Courrent A, Delachambre J (1992) cDNA cloning and deduced amino acid sequence of a major, glycine-rich cuticular protein from the coleopteran Tenebrio molitor. Temporal and spatial distribution of the transcript during metamorphosis. Eur J Biochem 206:813-819

Cobianchi F, Biamonti G, Maconi M, Riva S (1994) Human hnRNP protein A1: a model polypeptide for a structural and genetic investigation of a broad family of RNA binding proteins. Genetica 94:101-114

Colombani J, Raisin S, Pantalacci S, Radimerski T, Montagne J, Léopold P (2003) A nutrient sensor mechanism controls Drosophila growth. Cell 114:739-749

Futahashi R, Okamoto S, Kawasaki H, Zhong YS, Iwanaga M, Mita K, Fujiwara H (2008) Genome-wide identification of cuticular protein genes in the silkworm, Bombyx mori. Insect Biochem Mol Biol 38:1138-1146

Goberdhan DC, Meredith D, Boyd CA, Wilson C (2005) PAT-related amino acid transporters regulate growth via a novel mechanism that does not require bulk transport of amino acids. Development 132:2365-2375

Hamodrakas SJ, Etmektzoglou T, Kafatos FC (1985) Amino acid periodicities and their structural implications for the evolutionarily conservative central domain of some silkmoth chorion proteins. J Mol Biol 186:583-589

Højrup P, Andersen SO, Roepstorff P (1986) Isolation, characterization, and N-terminal sequence studies of cuticular proteins from the migratory locust, Locusta migratoria. Eur J Biochem 154:153-159

Horie Y, Inokuchi T (1978) Protein synthesis and uric acid excretion in the absence of essential amino aicds in the silkworm, Bombyx mori. Insect Biochem 8:251-254

Keller B (1993) Structural cell wall proteins. Plant Physiol 101:1127-1130

Ma S, Zhang S, Wang F, Liu Y, Liu Y, Xu H, Liu C, Lin Y, Zhao P, Xia Q (2012) Highly efficient and specific genome editing in silkworm using custom TALENs. PLoS One 7:e45035. doi:10.1371/ journal.pone.0045035

Mangeon A, Junqueira RM, Sachetto-Martins G (2010) Functional diversity of the plant glycine-rich proteins superfamily. Plant Signal Behav 5:99-104

Maruyama SR, Anatriello E, Anderson JM, Ribeiro JM, Brandão LG, Valenzuela JG, Ferreira BR, Garcia GR, Szabó MP, Patel S, Bishop R, de Miranda-Santos IK (2010) The expression of genes coding for distinct types of glycine-rich proteins varies according to the biology of three metastriate ticks, Rhipicephalus (Boophilus) microplus, Rhipicephalus sanguineus and Amblyomma cajennense. BMC Genom 11:363-379

Mehrel T, Hohl D, Rothnagel JA, Longley MA, Bundman D, Cheng C, Lichti U, Bisher ME, Steven AC, Steinert PM, Stuart H, Yirspa SH, Roop DR (1990) Identification of a major keratinocyte cell envelope protein, loricrin. Cell 61:1103-1112

Mounier N, Prudhomme JC (1986) Isolation of actin genes in Bombyx mori: the coding sequence of a cytoplasmic actin gene expressed in the silk gland is interrupted by a single intron in an unual position. Biochimie 68:1053-1056

Mousavi A, Hotta Y (2005) Glycine-rich proteins: a class of novel proteins. Appl Biochem Biotechnol 120:169-174

Park CJ, Park CB, Hong SS, Lee HS, Lee SY, Kim SC (2000) Characterization and cDNA cloning of two glycine- and histidine-rich antimicrobial peptides from the roots of shepherd's purse, Capsella bursa-pastoris. Plant Mol Biol 44:187-197

Sachetto-Martins G, Franco LO, de Oliveira DE (2000) Plant glycinerich proteins: a family or just proteins with a common motif? Biochim Biophys Acta 1492:1-14

Sato N (1994) A cold-regulated cyanobacterial gene cluster encodes RNA-binding protein and ribosomal protein S21. Plant Mol Biol 24:819-823 
Sudo S, Fujikawa T, Nagakura T, Ohkubo T, Sakaguchi K, Tanaka M, Nakashima K, Takahashi T (1997) Structures of mollusc shell framework proteins. Nature 387:563-564

Suzuki Y, Matsuoka T, Iimura Y, Fujiwara H (2002) Ecdysteroiddependent expression of a novel cuticle protein gene BMCPG1 in the silkworm, Bombyx mori. Insect cuticular proteins. Insect Biochem Mol Biol 32:599-607

Takasu Y, Sajwan S, Daimon T, Osanai-Futahashi M, Uchino K, Sezutsu H, Tamura T, Zurovec M (2013) Efficient TALEN construction for Bombyx mori gene targeting. PLoS One 18:e73458. doi:10.1371/journal.pone.0073458

Wullschleger S, Loewith R, Hall MN (2006) TOR signaling in growth and metabolism. Cell 124:471-484

Yanagawa H, Watanabe K, Hirayama C, Shinbo H (1995) Quantative requirment for amino acids and effects of dietary amino acid balance on growth in polyphagous strains of the silkworm, Bombyx mori. Bull Natl Inst Agric Sci (in Japanese) 15:63-83
Yemm E, Cocking EC (1954) Estimation of amino acids by nninhydrin. Biochem J 58((330th Meeting)):xii

Yoshizawa Y, Sato R, Tsuchihara K, Ozaki K, Mita K, Asaoka K, Taniai K (2011) Ligand carrier protein genes expressed in larval chemosensory organs of Bombyx mori. Insect Biochem Mol Biol 41:545-462

Zhang J, Goyer C, Pelletier Y (2008) Environmental stresses induce the expression of putative glycine-rich insect cuticular protein genes in adult Leptinotarsa decemlineata (Say). Insect Mol Biol 17:209-216

Zhong YS, Mita K, Shimada T, Kawasaki H (2006) Glycine-rich protein genes, which encode a major component of the cuticle, have different developmental profiles from other cuticle protein genes in Bombyx mori. Insect Biochem Mol Biol 36:99-110

Zhou S, Zhou Q, Liu Y, Wang S, Wen D, He Q, Wang W, Bendena WG, Li S (2010) Two Tor genes in the silkworm Bombyx mori. Insect Mol Biol 19:727-735 